\title{
STATISTICAL AVERAGE ESTIMATES OF HIGH LATITUDE FIELD-ALIGNED CURRENTS FROM THE STARE AND SABRE COHERENT VHF RADAR SYSTEMS
}

\author{
M. J. Kosch ${ }^{1}$ and E. Nielsen \\ Max-Planck-Institut für Aeronomie, D-37189 Katlenburg-Lindau, Germany \\ ${ }^{1}$ Now at Dept. Communication Systems, LA1 4YR Lancaster University,UK
}

\begin{abstract}
Two bistatic VHF radar systems, STARE and SABRE, have been employed to estimate ionospheric electric fields in the geomagnetic latitude range $61.1-69.3^{\circ}$ (geographic latitude range $63.8-72.6^{\circ}$ ) over northern Scandinavia. 173 days of good backscatter from all four radars have been analysed during the period 1982 to 1986, from which the average ionospheric divergence electric field versus latitude and time is calculated. The average magnetic fieldaligned currents are computed using an AE-dependent empirical model of the ionospheric conductance. Statistical Birkeland current estimates are presented for high and low values of the $\mathrm{Kp}$ and $\mathrm{AE}$ indices as well as positive and negative orientations of the IMF $\mathrm{B}_{\mathrm{z}}$ component. The results compare very favourably to other ground-based and satellite measurements.
\end{abstract}

\section{INTRODUCTION}

Magnetic field-aligned currents (FACs) at high latitudes provide the energy link coupling the ionosphere with the magnetosphere. There have been many studies of global FACs using satellite (e.g. Zmuda and Armstrong (1974), Iijima and Potemra (1976a, 1976b, 1978)) and ground-based data (e.g. Caudal (1987), Fontain and Peymirat (1996, 1997), Foster et al. (1989), Friis-Christensen et al. (1985), Kamide et al. (1981) and Yasuhara et al. (1982)). However, they were all based on rather small data sets. In this study we have used 173 days of data, taken during 1982 - 1986, to produce the global statistically averaged distribution of FACs for high latitudes. Most of the data comes from the equinoxes (Kosch and Nielsen, 1995). The FACs are computed from electric field data obtained from the STARE (Greenwald et al., 1978) and SABRE (Nielsen et al., 1983) coherent backscatter bistatic VHF radar systems in conjunction with the global ionospheric conductance model of Spiro et al. (1982). STARE covers a geographic area of $67.6-72.6^{\circ} \mathrm{N}$ and $13.5-26.0^{\circ} \mathrm{E}$, whilst SABRE covers $63.4-68.4^{\circ} \mathrm{N}$ and $0.5^{\circ} \mathrm{W}$ $12.5^{\circ} \mathrm{E}$. Details concerning the radars and the data set, including plots of the conductance model, have been presented by Kosch and Nielsen (1995). For this study, global FACs are computed in the combined radars' field of view of $61.1-69.3^{\circ}$ geomagnetic latitude using $0.2^{\circ}$ increments and 2 minutes steps $\left(0.5^{\circ}\right.$ longitude) in magnetic local time (MLT). The FACs are averaged in the overlapping part of the field of view.

\section{THEORY}

We derive the equation for field-aligned currents (FACs) in the ionosphere. Ohm's law in the ionosphere is:

$\mathbf{J}=\Sigma_{\mathrm{P}} \mathbf{E}+\Sigma_{\mathrm{H}}(\hat{\mathbf{B}} \times \mathbf{E})$ 
where $\mathbf{J}$ is the current vector, $\mathbf{E}$ the electric field vector, $\Sigma_{\mathrm{P}}$ and $\Sigma_{\mathrm{H}}$ are the Pedersen and Hall conductances, respectively, and $\hat{\mathbf{B}}$ is the unit vector in the direction of the earth's magnetic field. Assuming a vertical magnetic field with $\hat{\mathbf{B}}$ pointing along the Z-axis, $\mathbf{\imath}$ being the unit vector pointing downwards, the FAC $\left(\mathrm{J}_{\|}\right)$is given by:

$\mathbf{J}_{\|}=\operatorname{div} \mathbf{J}=\operatorname{div}\left(\Sigma_{\mathrm{P}} \mathbf{E}\right)+\operatorname{div}\left(\Sigma_{\mathrm{H}}(\hat{\mathbf{Z}} \times \mathbf{E})\right)$

Expanding Eq. 2,

$\mathrm{J}_{\|}=\Sigma_{\mathrm{P}} \operatorname{div} \mathbf{E}+\nabla \Sigma_{\mathrm{P}} \bullet \mathbf{E}+\nabla \Sigma_{\mathrm{H}} \bullet(\hat{\mathbf{z}} \times \mathbf{E})+\Sigma_{\mathrm{H}} \operatorname{div}(\hat{\mathbf{z}} \times \mathbf{E})$

The last term of Eq. 3 is zero because $\operatorname{div}(\hat{\mathbf{z}} \times \mathbf{E})=\mathbf{E} \bullet \operatorname{rot}(\hat{\mathbf{z}})-\hat{\mathbf{z}} \bullet \operatorname{rot}(\mathbf{E}) \quad$ where $\mathbf{E} \bullet \operatorname{rot}(\hat{\mathbf{z}})=0$ by definition and $\hat{\mathbf{Z}} \bullet \mathbf{r o t}(\mathbf{E})=0$ since we assume that a curl-free potential electric field exists in the ionosphere (ie. only slow time variations). Hence:

$\mathbf{J}_{\|}=\Sigma_{\mathrm{P}} \operatorname{div} \mathbf{E}+\nabla \Sigma_{\mathrm{P}} \bullet \mathbf{E}+\nabla \Sigma_{\mathrm{H}} \bullet(\mathbf{z} \times \mathbf{E})$

Neglecting conductance gradients:

$\mathrm{J}_{\|} \approx \Sigma_{\mathrm{P}} \operatorname{div} \mathbf{E}$

Assuming uniform conductances (i.e. dropping the last 2 terms of Eq. 4) may introduce uncertainties in computing the FACs. However, in a statistically averaged sense, it is expected that term 1 will dominate. Smallscale conductance gradients and electric field variations due to auroral precipitation (e.g. Kosch et al. (1998), Nielsen and Kosch (1999)) should effectively be averaged away. Large-scale gradients are generally small on the grid size used here.

The practical computation of div $\mathbf{E}$ involves integrating the perpendicular component of the electric field vector around a closed loop. In this case, we use squares corresponding to the grid step size of the data. This means that 4 electric field vectors, one on each side of the square, contribute to a single FAC value. If any one electric field vector is missing, then the FAC for that grid point is not computed.

\section{RESULTS}

The radars receive most of their backscatter for $2<\mathrm{Kp}<7$ (Kosch and Nielsen, 1995). Figure 1 shows the global statistically averaged horizontal Pedersen currents for low (Kp $<4$, left panel) and high (Kp $>4$, right panel) geomagnetic activity. The latitude scale is linear with the inner and outer circles representing $75^{\circ}$ and $60^{\circ}$ of geomagnetic latitude, respectively. The current flow pattern is similar to the well known convection electric field pattern with the Harang discontinuity visible around 22 MLT. Clearly, the vector amplitudes are modulated by the Pedersen conductance. The FACs are computed by taking the divergence of these vectors, assuming negligible conductance gradients, and are shown in Figure 2 in the same format as Figure 1. The entire post midday sector up to about 20 MLT is dominated by downward current, mostly with current densities of $<1 \mu \mathrm{A} / \mathrm{m}^{2}$. Between approximately 23 and 06 MLT there is a region of strong downward current on the poleward edge of the field of view, with current densities exceeding $2 \mu \mathrm{A} / \mathrm{m}^{2}$ in places. The remainder of the field of view consists of upward current, with current densities up to $2 \mu \mathrm{A} / \mathrm{m}^{2}$.

For high geomagnetic activity, a region of strong downward current appears in the middle of the latitude range between 0 and 5 MLT. We believe this to be an artefact which is probably due to strong conductance gradients which would violate our initial assumption. Since this feature only appears at high geomagnetic activity in the postmidnight sector, it is probably associated with auroral precipitation which would produce such an effect. Ignoring the artefact, the morphology and FAC densities in our plots compare very well to previous studies (e.g. Caudal (1987), Fontaine and Peymirat (1996), Foster et al. (1989), Iijima and Potemra (1976a, 1976b, 1978), Kamide et al. (1981), Yasuhara et al. (1982), Zmuda and Armstrong (1974)). The entire FAC pattern rotates to approximately one hour earlier with increased geomagnetic activity. 
Although the net total current should be zero, with global upward and downward FACs exactly cancelling, for $\mathrm{Kp}<4$ and $\mathrm{Kp}>4$ there is a net upward current of $1.01 \times 10^{6}$ and $0.82 \times 10^{6} \mathrm{~A}$, respectively. This results from our field of view not going to high enough latitudes to completely encompass the poleward downward currents.

Since STARE and SABRE cover the auroral zone, the AE index is a more suitable way of looking for the effects of geomagnetic activity than the global Kp index discussed above. Figure 3 shows the global average FACs for AE $<500$ (left panel) and AE > 500 (right panel) in the same format as Figure 2. The net upward currents are $1.24 \mathrm{x}$ $10^{6}$ and $0.64 \times 10^{6} \mathrm{~A}$, respectively. The comments made above for Figure 2 also apply to these figures.

Magnetic reconnection between the interplanetary magnetic field (IMF) and the earth's magnetic field occurs for the IMF component $\mathrm{Bz}<0$ which is usually associated with increased geomagnetic activity. Figure 4 is the same as Figure 2 but for $\mathrm{Bz}>0$ (left panel) and $\mathrm{Bz}<0$ (right panel) with net upward currents of $0.92 \times 10^{6}$ and $0.1 \times 10^{6} \mathrm{~A}$, respectively. Again, the comments made above for Figure 2 apply to these figures.

The total upward FAC strength within the entire field of view, corresponding mostly to Region 2 (equatorward) currents, has been computed versus the geomagnetic activity indices and are given in Table 1 in mega-Amperes. The total downward current has not been computed since the field of view does not extend poleward sufficiently to completely include these currents and the FAC artefact, mentioned earlier, consists entirely of downward FACs. Clearly, the total upward FAC strength increases with increasing $\mathrm{Kp}, \mathrm{AE}$ and negative Bz. The FAC strength as a function of $\mathrm{Bz}$ has been split into $\mathrm{Bz}<0$ and $\mathrm{Bz}>0$ ranges due to the very non-linear response of the $\mathrm{FAC}$ as $\mathrm{Bz}$ changes sign. Curve fitting results in the following relationships:
$\mathrm{FAC}_{\mathrm{up}}=1.401+6.01 \times 10^{-3} \mathrm{AE}$
$\mathrm{FAC}_{\mathrm{up}}=1.658+0.054 \mathrm{Kp}+0.102 \mathrm{Kp}^{2}$
mega-Amperes, for $\mathrm{AE}$
mega-Amperes, for $\mathrm{Kp}$
$\mathrm{FAC}_{\mathrm{up}}=3.437-0.412 \mathrm{Bz}$
mega-Amperes, for $\mathrm{Bz}<0$
$\mathrm{FAC}_{\text {up }}=3.588-0.546 \mathrm{Bz}+0.227 \mathrm{Bz}^{2}-0.029 \mathrm{Bz}^{3}$
mega-Amperes, for $\mathrm{Bz}>0$

These results are consistent with the findings of Iijima and Potemra (1976a). For AE and Kp equal to zero, there is still a considerable total upward FAC of 1.4 and 1.66 mega-Amperes, respectively. Hence, even for no geomagnetic forcing there still appears to be some ionosphere-magnetosphere coupling. The total upward FAC decreases with increasingly positive Bz, which is consistent with the results of Foster et al. (1989). Additionally, there seems to be a distinct cut off in the FAC for $\sim \mathrm{Bz}>6$. For large positive values of $\mathrm{Bz}$, coupling of the magnetosphere to the ionosphere is almost stopped when magnetic reconnection between the magnetosphere and the IMF is also severely reduced. The two equations for $\mathrm{Bz}$ are almost, but not exactly, equal for $\mathrm{Bz}=0$, however, the result is still rather good. Clearly, $\mathrm{FAC}_{\mathrm{up}}$ becoming negative for large $\mathrm{Bz}$ positive is most probably unrealistic.

Table 1. Total upward FAC versus $\mathrm{Kp}, \mathrm{AE}$ and $\mathrm{Bz}$ in mega-Amperes

\begin{tabular}{|c|c|c|c|c|c|}
\hline Kp & FAC & AE & FAC & Bz & FAC \\
\hline $0-2$ & 1.77 & $0-200$ & 2.19 & $<-8$ & 7.17 \\
\hline $2-3$ & 2.50 & $200-400$ & 3.07 & $-8--6$ & 6.21 \\
\hline $4-5$ & 3.13 & $400-600$ & 4.08 & $-6--4$ & 5.82 \\
\hline $5-6$ & 4.76 & $600-800$ & 5.91 & $-4--2$ & 4.46 \\
\hline $6-7$ & 6.50 & $800-1000$ & 6.78 & $-2-0$ & 3.87 \\
\hline$>7$ & 9.50 & $>1000$ & 7.89 & $0-2$ & 3.24 \\
\hline & & & & $2-4$ & 3.20 \\
\hline & & & & $4-6$ & 2.86 \\
\hline & & & & $6-8$ & 0.81 \\
\hline & & & & $>8$ & 0.37 \\
\hline
\end{tabular}

\section{CONCLUSIONS}

The global average pattern of Birkeland currents as seen by the STARE and SABRE radars is in generally good agreement with previous studies, even though no account for conductance gradients is included here. There appears to be a small net average upward current $\left(<0.1 \mu \mathrm{A} / \mathrm{m}^{2}\right)$ within the radars' combined field of view which persists for 
all geomagnetic conditions. This is due to downward FACs in the post-midnight sector which are poleward of $70^{\circ}$ and outside of the radars' combined field of view. The evening transition from Region 1 (poleward) upward FAC to Region 1 downward FAC (equivalently, Region 2 (equatorward) downward FAC to Region 2 upward FAC) occurs about one hour earlier for high $\mathrm{Kp}$, high $\mathrm{AE}$ and $\mathrm{Bz}<0$ than is the case for low $\mathrm{Kp}$, low $\mathrm{AE}$ and $\mathrm{Bz}>0$, respectively.

There is a clear monotonic increase in FAC strength with increasing geomagnetic activity, indicative of increased coupling between the ionosphere and magnetosphere. This relationship appears to be linear in AE and quadratic in Kp. For large positive $\mathrm{Bz}$ values the FACs are virtually turned off indicating that magnetic reconnection between the IMF and the magnetosphere is also greatly reduced.

It is the authors' opinion that the downward current feature seen at approximately 0 - 5 MLT in the middle of the combined field of view results from neglecting the conductance gradients, ie. terms 2 and 3 of Eq. 4. This is supported by the fact that this artefact virtually disappears for low geomagnetic activity and only occurs in the region where the strongest auroral precipitation occurs. Preliminary analysis supports this conjecture. Conductance gradients will be included in a future study.

\section{REFERENCES}

Caudal, G., Field-Aligned Currents Deduced from EISCAT Radar Observations and Implications Concerning the Mechanism that Produces Region 2 Currents, J. Geophys. Res., 92, 6000, 1987.

Fontaine, D., and C. Peymirat, Large-scale distributions of ionospheric horizontal and field-aligned currents inferred from EISCAT, Ann. Geophys., 14, 1284, 1996.

Fontaine, D., and C. Peymirat, The large-scale current system and the ionosphere/magnetosphere coupling, Adv. Space Res., 20(3), 459, 1997.

Foster, J. C., T. Fuller-Rowell and D. S. Evans, Quantitative Patterns of Large-Scale Field-Aligned Currents in the Auroral Ionosphere, J. Geophys. Res., 94, 2555, 1989.

Friis-Christensen, E., Y. Kamide, A. D. Richmond and S. Matsushita, Interplanetary Magnetic Field Control of High-Latitude Electric Fields and Currents Determined from Greenland Magnetometer Data, J. Geophys. Res., 90, 1325, 1985.

Greenwald, R. A., W. Weiss, E. Nielsen and N. R. Thomson, STARE: A new radar auroral backscatter experiment in northern Scandinavia, Radio Sci., 13, 1021, 1978.

Iijima, T., and T. A. Potemra, The Amplitude Distribution of Field-Aligned Currents at Northern High Latitudes Observed by TRIAD, J. Geophys. Res., 81, 2165, 1976a.

Iijima, T., and T. A. Potemra, Field-Aligned Currents in the Dayside Cusp Observed by Triad, J. Geophys. Res., 81, 5971, 1976b.

Iijima, T., and T. A. Potemra, Large-Scale Characteristics of Field-Aligned Currents Associated with Substorms, J. Geophys. Res., 83, 599, 1978.

Kamide, Y., A. D. Richmond and S. Matsushita, Estimation of Ionospheric Electric Fields, Ionospheric Currents, and Field-Aligned Currents From Ground Magnetic Records, J. Geophys. Res., 86, 801, 1981.

Kosch, M. J., and E. Nielsen, Coherent radar estimates of average high-latitude ionospheric Joule heating, $J$. Geophys. Res., 100, 12201, 1995.

Kosch, M. J., M. W. J. Scourfield and E. Nielsen, A self-consistent explanation for a plasma flow vortex associated with the brightening of an auroral arc, J. Geophys. Res., 103, 29383, 1998.

Nielsen, E., W. Güttler, E. C. Thomas, C. P. Stewart, T. B. Jones and A. Hedberg, A new radar auroral backscatter experiment, Nature, 304, 712, 1983.

Nielsen, E., and M. J. Kosch, Joint observations with STARE and DASI, Proceedings of the 25th Annual European Meeting (1998) on Atmospheric Studies by Optical Methods in Granada, Spain, 62, 1999.

Spiro, R. W., P. H. Reiff, and L. J. Maher Jr., Precipitating electron energy flux and auroral zone conductance: An empirical model, J. Geophys. Res., 87, 8215, 1982.

Yasuhara, F., Y. Kamide and J. M. Holt, Field-aligned currents in high latitudes estimated from Millstone Hill radar observations of ion drifts, J. Geophys. Res., 87, 2553, 1982.

Zmuda, A. J., and J. C. Armstrong, The Diurnal Flow Pattern of Field-Aligned Currents, J. Geophys. Res., 79, 4611, 1974. 

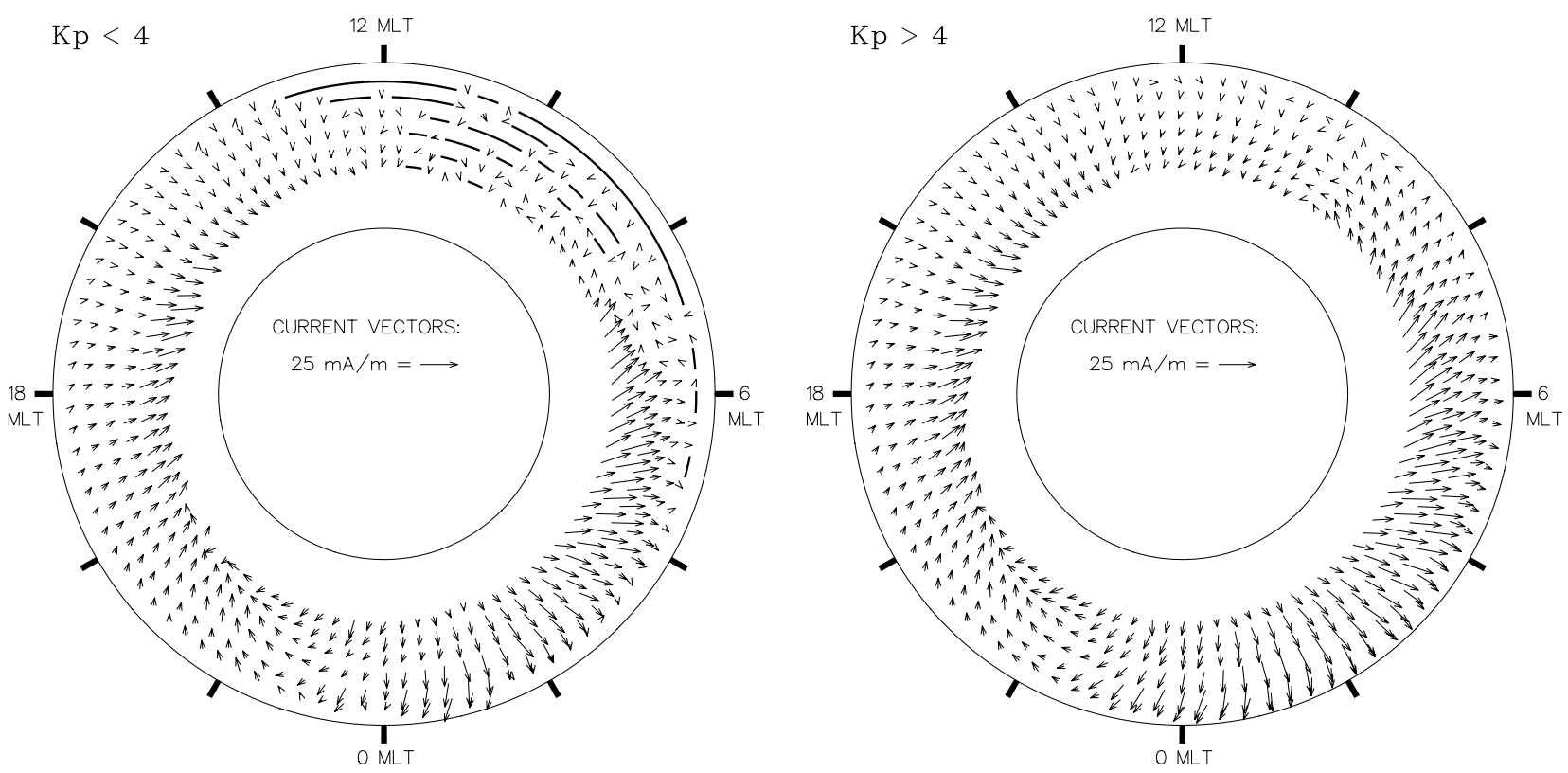

Fig. 1. Average horizontal Pedersen currents for the geomagnetic latitude range of $61.1-69.3^{\circ}$ as a function of magnetic local time. The left and right panels are for $\mathrm{Kp}<4$ and $\mathrm{Kp}>4$, respectively. The curved line segments indicate no data.
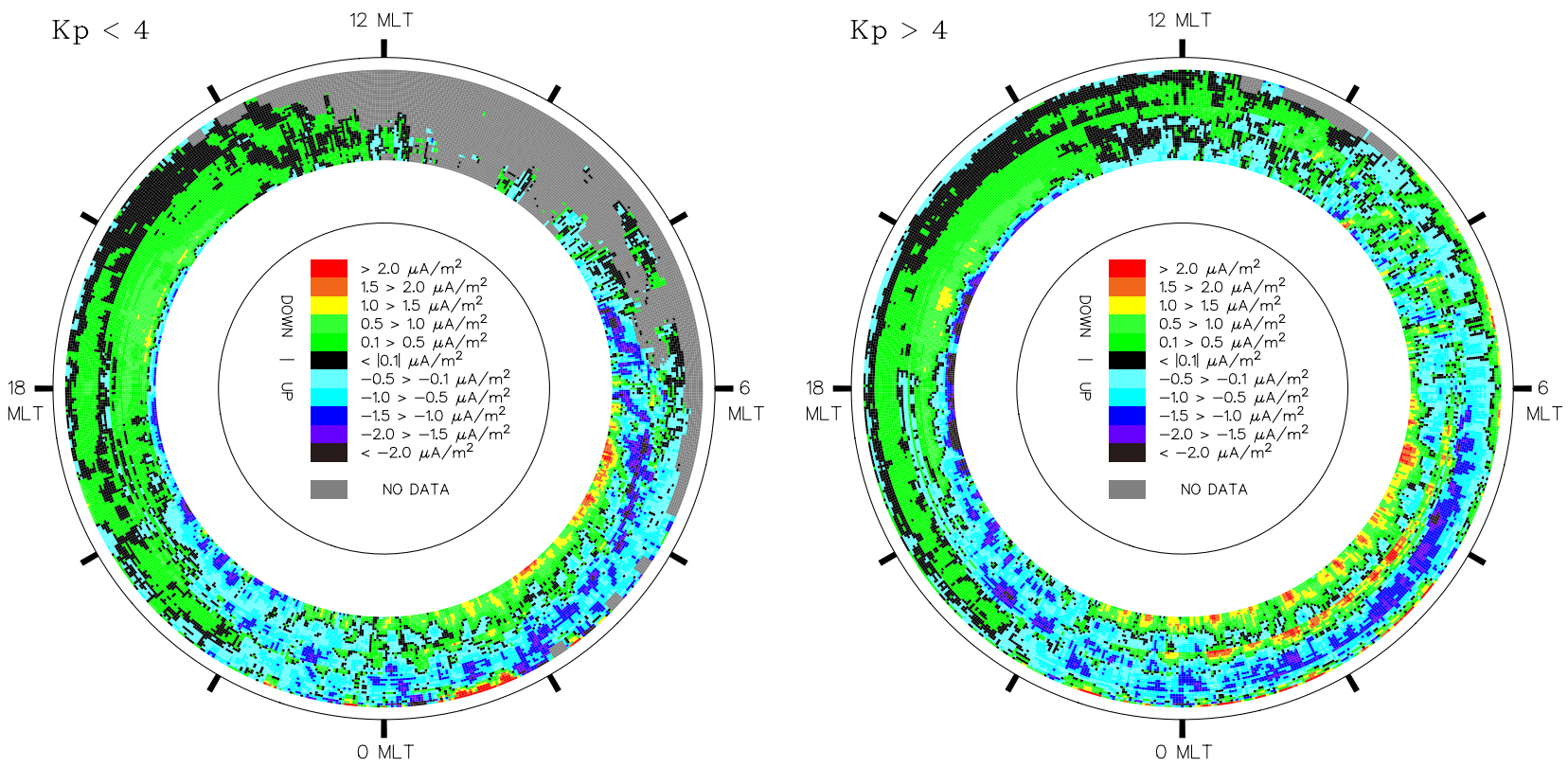

Fig. 2. Average field-aligned currents for the geomagnetic latitude range of $61.2-69.2^{\circ}$ as a function of magnetic local time. The left and right panels are for $\mathrm{Kp}<4$ and $\mathrm{Kp}>4$, respectively. 

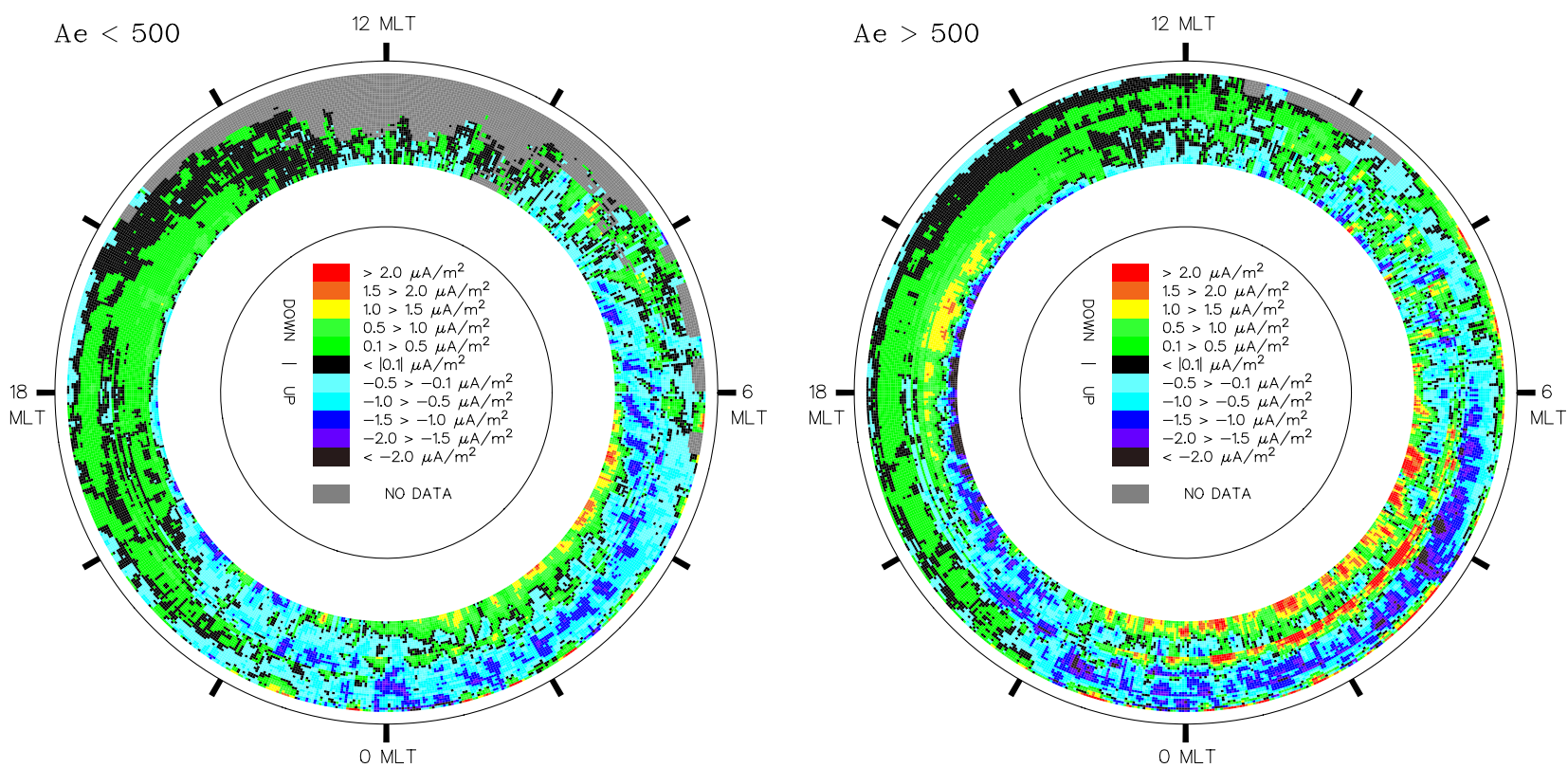

Fig. 3. Average field-aligned currents for the geomagnetic latitude range of $61.2-69.2^{\circ}$ as a function of magnetic local time. The left and right panels are for $\mathrm{AE}<500$ and $\mathrm{AE}>500$, respectively.
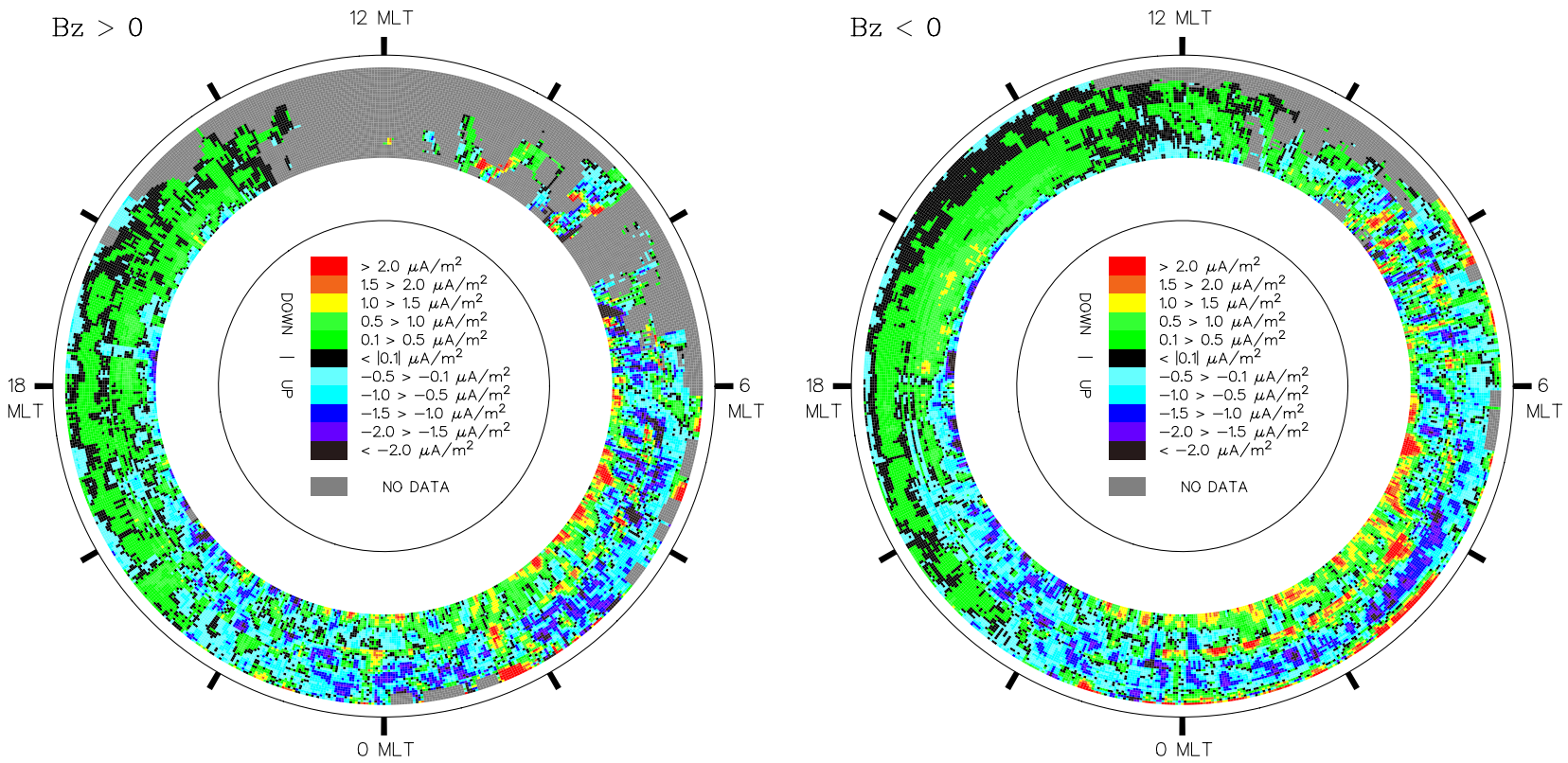

Fig. 4. Average field-aligned currents for the geomagnetic latitude range of $61.2-69.2^{\circ}$ as a function of magnetic local time. The left and right panels are for $\mathrm{Bz}>0$ and $\mathrm{Bz}<0$, respectively. 